\title{
On supplementary information
}

Nature Immunology is implementing new editorial guidelines for the supplementary material of research articles.

$\mathrm{B}$ eginning with this issue, Nature Immunology is changing its guidelines for supplementary information by implementing a restriction on the number of supplementary figures that accompany research articles. Under these new guidelines, the number of supplementary figures should not exceed the number of main figures in each article. This change represents a natural response to feedback received during the past year from the research community. Immunologists, along with researchers in other areas of science, are unsatisfied with the present practice of adding an ever-growing amount of supplementary information to published papers.

A change is needed in the way supplementary material is viewed. Authors often complain that experimental data requested during the review process and requiring substantial effort to generate, in terms of time and money, ends up as part of a large file of supplementary information that the non-aficionado might never access. Indeed, this complaint may be part of a more general dissatisfaction with the present tendency of the review process to always demand additional data, some only marginally related to the main story. For referees, a large amount of supplementary data makes it more difficult to find the information needed to evaluate manuscripts, adds considerably to the total workload and possibly increases the time required for review. In addition, readers complain that authors sometimes use the supplementary information section as a repository for unrelated and unpublished data in search of a home.

Those are all justified concerns that are only part of a more complex picture. The size of the average research article has continued to grow over time, and as more data are included in one report, more controls and additional experimental clarifications must be presented along with the main results. We recognize the utility of having a repository for this additional information. Data that provide proof of principle of the utility of an assay or that demonstrate efficient deletion of a protein or silencing of an RNA message, experiments that exclude alternative possibilities, and gating strategies for complex flow cytometry or sorting procedures represent an important and informative part of the research that needs to be presented to the audience. They provide experts in the field and reviewers with valuable details on how the experiments were done and how conclusions and interpretations were derived from the data. In addition, certain types of data, such as movies, imaging files or large data sets, can be included only as supplementary information and thus will continue to have no restric- tions. Consistent with that, we will allow resource articles, which are reports of large data sets, such as comprehensive analyses of proteins or mRNA in various cells or tissues, to have an unlimited number of associated supplementary items. However, the same guiding principle should be applied when deciding what to display in the supplementary information or main body of a manuscript; that is, data that are directly relevant to the main conclusions of the paper.

As always, the main purpose of the review and editorial processes here at Nature Immunology is not only to report innovative and technically reliable results but also to produce a coherent, elegant and selfcontained body of work. We encourage authors to be selective and carefully review the content and extent of the supplementary material included in research articles at submission. As the review process might result in the addition of new data, the editors will work with the authors and reviewers to critically evaluate the scope and quality of the supplementary information. During the editing stage, we will continue to provide help and suggestions to ensure that this new material is sensibly integrated into the manuscript and that important information is easily available to our readers.

As part of the same effort to streamline our papers and to make them more accessible to our readers, supplementary methods will no longer be included in the supplementary information. All research and resource papers will have a single methods section included with the main body of the article, but it will be published online only. To accommodate this change, we have increased the size of the methods section from the previous limit of 1,000 words to a limit of approximately 1,500 words. On a case-by-case basis, longer methods sections will be accepted at the editor's discretion. To compensate for limitations in the length of the methods section, Nature Protocols offers the option of uploading protocols used for each manuscript to Protocol Exchange, an open online resource that allows researchers to share detailed protocols and experimental know-how. Protocols are linked from the article, are made freely available, are assigned digital object identifier numbers for ease of citation and are fully searchable at the Nature website.

Less can be more, and by imposing restrictions on the number of supplementary figures and reorganizing the methods section, we hope to shape more concise and focused papers that will benefit our audience. As needs may change in the future, the guidelines for and format of our research articles will change as well, in an effort to accommodate the requirements of quickly evolving research. 